\title{
Higher versus Lower Oxygen Concentration during Respiratory Support in the Delivery Room in Extremely Preterm Infants: A Pilot Feasibility Study
}

\author{
Brenda Hiu Yan Law ${ }^{1,2}{ }^{\circledR}$, Elizabeth Asztalos ${ }^{3}$, Neil N. Finer ${ }^{4,5}$, Maryna Yaskina ${ }^{6}$, Maximo Vento ${ }^{7,8,9} \oplus$, \\ William Tarnow-Mordi ${ }^{10}$, Prakesh S. Shah ${ }^{11}$ and Georg M. Schmölzer ${ }^{1,2, *(1)}$
}

1 Centre for the Studies of Asphyxia and Resuscitation, Neonatal Research Unit, Royal Alexandra Hospital, Alberta Health Services, Edmonton, AB T5H 3V9, Canada; blaw2@ualberta.ca

2 Division of Neonatology, Department of Pediatrics, University of Alberta, Edmonton, AB T6G 2R3, Canada

3 Sunnybrook Health Sciences Centre, Department of Paediatrics, University of Toronto,

Toronto, ON M4N 3M5, Canada; elizabeth.asztalos@sunnybrook.ca

4 School of Medicine, University of California, San Diego, CA 92093, USA; nfiner@health.ucsd.edu

5 Sharp Mary Birch Hospital for Women and Newborns, San Diego, CA 92123, USA

6 Women and Children's Health Research Institute, Department of Pediatrics, University of Alberta, Edmonton, AB T6G 1C9, Canada; yaskina@ualberta.ca

7 Health Research Centre, University and Polytechnic Hospital La Fe, 46026 Valencia, Spain; maximo.vento@uv.es

8 Division of Neonatology, University and Polytechnic Hospital La Fe, 46026 Valencia, Spain

9 Spanish Maternal and Infant Health and Development Network, Health Research Institute Carlos III, National Network, 46026 Madrid, Spain

check for
updates

Citation: Law, B.H.Y.; Asztalos, E. Finer, N.N.; Yaskina, M.; Vento, M.; Tarnow-Mordi, W.; Shah, P.S.;

Schmölzer, G.M. Higher versus Lower Oxygen Concentration during Respiratory Support in the Delivery Room in Extremely Preterm Infants: A Pilot Feasibility Study. Children 2021, 8, 942. https://doi.org/ $10.3390 /$ children 8110942

\section{Academic Editor: Sari A. Acra}

Received: 8 September 2021

Accepted: 15 October 2021

Published: 20 October 2021

Publisher's Note: MDPI stays neutral with regard to jurisdictional claims in published maps and institutional affiliations.

Copyright: (c) 2021 by the authors. Licensee MDPI, Basel, Switzerland. This article is an open access article distributed under the terms and conditions of the Creative Commons Attribution (CC BY) license (https:/ / creativecommons.org/licenses/by/ $4.0 /)$.
10 NHMRC Clinical Trials Centre, University of Sydney, Camperdown, NSW 2050, Australia; wotarnowmordi@gmail.com

11 Department of Pediatrics, Mount Sinai Hospital, University of Toronto, Toronto, ON M5G 1X5, Canada; prakeshkumar.shah@sinaihealth.ca

* Correspondence: georg.schmoelzer@me.com; Tel.: +1-780-735-4647; Fax: +1-780-735-4072

Abstract: Background: Optimal starting oxygen concentration for delivery room resuscitation of extremely preterm infants (<29 weeks) remains unknown, with recommendations of $21-30 \%$ based on uncertain evidence. Individual patient randomized trials designed to answer this question have been hampered by poor enrolment. Hypothesis: It is feasible to compare $30 \%$ vs. $60 \%$ starting oxygen for delivery room resuscitation of extremely preterm infants using a change in local hospital policy and deferred consent approach. Study design: Prospective, single-center, feasibility study, with each starting oxygen concentration used for two months for all eligible infants. Population: Infants born at $23+0-28+6$ weeks' gestation who received delivery room resuscitation. Study interventions: Initial oxygen at $30 \%$ or $60 \%$, increasing by $10-20 \%$ every minute for heart rate $<100 \mathrm{bpm}$, or increase to $100 \%$ for chest compressions. Primary outcome: Feasibility, defined by (i) achieving difference in cumulative supplied oxygen concentration between groups, and (ii) post-intervention rate consent $>50 \%$. Results: Thirty-four infants were born during a 4-month period; consent was obtained in $63 \%$. Thirty ( $n=12,30 \%$ group; $n=18,60 \%$ group) were analyzed, including limited data from eight who died or were transferred before parents could be approached. Median cumulative oxygen concentrations were significantly different between the two groups in the first $5 \mathrm{~min}$. Conclusion: Randomized control trial of $30 \%$ or $60 \%$ oxygen at the initiation of resuscitation of extremely preterm neonates with deferred consent is feasible. Trial registration: Clinicaltrials.gov NCT03706586

Keywords: infant; newborn; delivery room; neonatal resuscitation; oxygen concentration

\section{Introduction}

In the minutes following birth, normal oxygen saturations $\left(\mathrm{SpO}_{2}\right)$ can be as low as $30 \%$ [1], which then increases to $85-95 \%$ over the next 7-10 $\mathrm{min}$ [2]. In term infants, resuscitation measures such as mask ventilation and supplemental oxygen may not be required to 
facilitate this transition. In contrast, most extremely preterm infants ( $<29$ weeks' gestation) will require respiratory support at birth [3]. In the past, preterm infants were resuscitated with $100 \%$ oxygen; however, in 2010 , neonatal resuscitation guidelines recommended air or "less oxygen" as initial oxygen concentrations, which should be then be adjusted to meet age-dependent $\mathrm{SpO}_{2}$ targets [4]. Since then, have clinicians mostly transitioned to a lower starting oxygen concentration strategy in the resuscitation of preterm infants [5].

The most recent neonatal resuscitation guidelines published in 2020 recommend starting oxygen concentrations of $21-30 \%$, based on uncertain evidence [6]. There is a lack of evidence for either overall benefit or harm in starting resuscitation with either lower $(<30 \%)$ or higher $(>65 \%)$ oxygen for preterm infants (i.e., $<37$ weeks' gestation) [6,7]. Indeed, a recent survey of 630 clinicians from 25 countries showed that the majority would initiate preterm infant delivery room stabilization with $30-40 \%$ oxygen [5]. The balance is between the harms of hypoxia vs. hyperoxia. On one hand, hyperoxia may lead to the generation of oxygen free radicals, increased oxidative stress, and end-organ damage. Hyperoxia may also alter cerebral blood flow [8-10]. On the other hand, hypoxia may also result in harms such as brain injury and death [8-10]; failure to achieve oxygen saturation $>80 \%$ at 5 min after birth has been associated with increased risk of IVH and death in both retrospective and prospective studies [11-13].

Randomized controlled trials and systematic reviews have evaluated different starting oxygen concentrations for resuscitation of preterm infants for the past 25 years, with varying definitions for "high" vs. "low" oxygen concentrations [7,13-15]. The conclusions for each trial and systematic review differ, highlighting the ongoing knowledge gap in this area. An individual patient meta-analysis by Oei et al. reported no difference in the overall risk of death with either lower $(\leq 30 \%)$ or higher $(\geq 60 \%)$ oxygen concentrations [14]. However, opposing results were seen in masked vs. unmasked trials, which the authors state could have represented a Type 1 error [14]. Furthermore, these meta-analyses also noted no differences in other common preterm morbidities when comparing low and high oxygen concentrations. [7,14] Finally, even with international cooperation, individual patient randomized controlled trials have had difficulty achieving target enrolment due to factors such as missed opportunities and clinicians declining to participate due to a perceived lack of equipoise $[15,16]$.

Despite advances in perinatal and neonatal care, neonates remain susceptible to oxidative and deleterious effects from hyperoxia and hypoxia [8-10]. There is a need for large, multi-center international trials of sufficient sample size, using an alternate recruitment strategy, powered to look at both safety outcomes such as mortality and longterm outcomes such as neurodevelopment. In preparation for such a trial, to ensure that we can achieve a difference in supplied oxygen between the two intervention groups and that we can obtain an acceptable rate of enrolment, we performed an unblinded prospective, single-center feasibility study of $30 \%$ vs. $60 \%$ starting oxygen concentration at birth in extremely preterm infants to determine the feasibility of a multi-centered clusterrandomized crossover design using deferred consent.

\section{Methods}

This was a prospective, single-center, feasibility study comparing two starting oxygen concentrations $\left(30 \% \mathrm{O}_{2}\right.$ vs. $\left.60 \% \mathrm{O}_{2}\right)$ during initial respiratory support at birth. Between November 2018 and February 2019, all eligible infants born between $23+0$ and $28+6$ weeks' gestation were included in the study. This feasibility study followed the design of a proposed cluster, crossover, randomized controlled trial with both interventions being implemented (Figure 1) using the CONSORT (Consolidated Standards of Reporting Trials) extension to randomized pilot and feasibility trials [17]. For this feasibility study, infants were managed at delivery with the first intervention $\left(30 \%\right.$ initial $\left.\mathrm{FiO}_{2}\right)$ for 2 months and then with the second intervention $\left(60 \%\right.$ initial $\left.\mathrm{FiO}_{2}\right)$ for another 2 months. This design mimicked a single-center participating in a cluster-randomized cross-over trial, where the local hospital policy would be changed to one of two randomized starting oxygen 
concentrations for a set recruitment period, with a similar pre-specified oxygen titration strategy for the entire trial duration. The study was carried out at the Royal Alexandra Hospital, Edmonton, a tertiary perinatal center admitting more than 350 infants with a birth weight of $<1500 \mathrm{~g}$ to the neonatal intensive care unit (NICU) annually. The Royal Alexandra Hospital Research Committee and Health Ethics Research Board, University of Alberta (Pro00084090) approved the study and the study was registered at Clinicaltrials.gov (NCT03706586) [18].

\subsection{Inclusion and Exclusion Criteria}

Inborn infants between $23+0$ and $28+6$ weeks' postmenstrual age were included in this study. Infants were excluded if they were (i) outborn (i.e., initial resuscitation not performed at the study center), (ii) born with a major congenital abnormality (e.g., congenital abnormalities that may affect oxygenation or neurodevelopmental outcomes), (iii) decision not to provide full resuscitation at birth, and (iv) if their parents declined to give consent after the study intervention.

\subsection{Consent}

We used a deferred consent approach, where written informed consent for use of patient data was sought from the parents as soon as possible after the birth after the initial resuscitation was completed. Further, our research ethics board approved limited data collection (i.e., delivery room data, in-hospital death, and major hospital morbidities) of infants for whom we did not have a chance to obtain parental consent due to death or being transferred to another facility within $72 \mathrm{~h}$ after birth. This approach allowed us to collect data for most infants receiving the intervention to allow the primary outcomes to be ascertained in all infants participating in the study.

\subsection{Sample Size Calculation}

For this pilot study, no sample size calculation has been performed. A convenient sample size of all infants within a 2-month time frame for each starting oxygen concentration group were recruited to determine the feasibility for recruitment for this intervention by using a cluster-randomized cross-over approach.

\subsection{Blinding}

Blinding was not feasible, as the first study intervention policy was assigned for two months and then switched to the alternate policy for another two months. However, the analysis team was blinded to group allocation.

\subsection{Study Interventions}

Delayed cord clamping for up to $60 \mathrm{~s}$ was attempted as per local hospital policy in all eligible infants. Other than starting oxygen concentration and oxygen titration strategy, all interventions such as mask ventilation, continuous positive airway pressure, intubation for poor respiratory effort or low heart rate, chest compressions, prevention of hypothermia by wrapping the infant in a polyethylene bag, and provision of appropriate medications were per the 2015 Neonatal Resuscitation Program guidelines. [7,8] The endotracheal intubation for the sole purpose of prophylactic surfactant administration was not allowed in the first $10 \mathrm{~min}$ after birth. After the first $10 \mathrm{~min}$, ongoing $\mathrm{SpO}_{2}$ targeting and neonatal care were provided according to our center's standard of care for both groups. 
(is) Hi-Lo Trial

Delayed cord clamping as per hospital policy

\section{Hi-Lo Pilot-Trial Algorithm}

From birth to $5 \mathrm{~min} \quad$ Eligible Infants: focus on effective respiratory support
$23^{0}-28^{6}$ weeks without congenital malformations and receiving full resuscitation

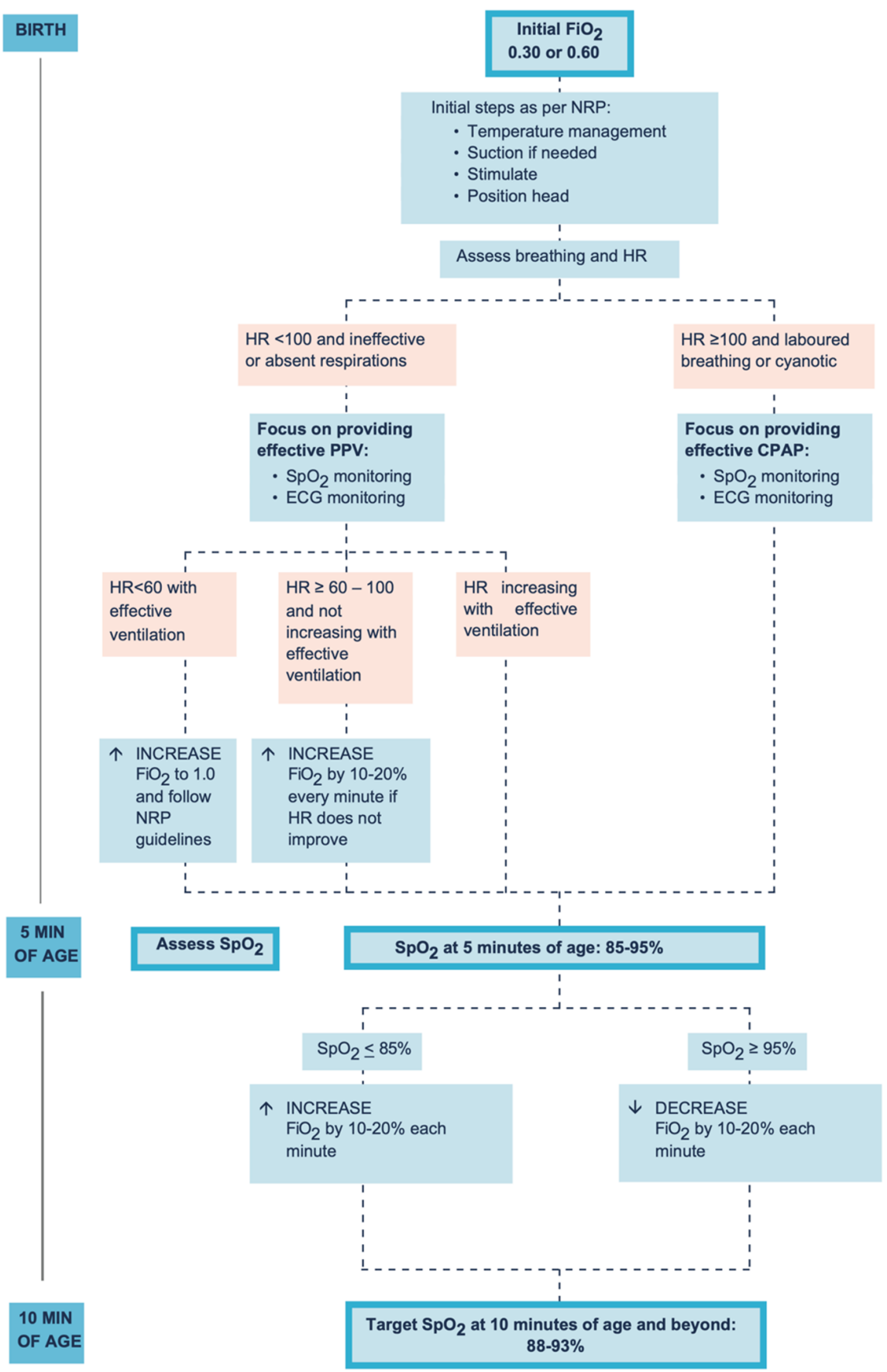

Figure 1. Study Interventions Flowchart.

Infants remained in $30 \%$ or $60 \% \mathrm{O}_{2}$ until 5 min of age unless the infant's heart rate (HR) remained $\leq 100$ beats per minute (bpm) and did not show a tendency towards progressive increase before reaching $5 \mathrm{~min}$ of age (oxygen concentration could then be increased by 
$10-20 \%$ every minute), or infant needs chest compression and/or epinephrine (oxygen concentration could then be increased to 100\%) (Figure 1). No alterations in oxygen concentration were made for an infant who was responding to resuscitation efforts with $\mathrm{HR}$ progressively increasing. At 5 min of age, the clinical team assessed $\mathrm{SpO}_{2}$ : If $\mathrm{SpO}_{2}$ was $\leq 85 \%$, oxygen was increased by $10-20 \%$ every 60 s to achieve $\mathrm{SpO}_{2}$ of $90-95 \%$ at $10 \mathrm{~min}$. If $\mathrm{SpO}_{2}$ was $\geq 95 \%$ oxygen was decreased (every 60 s) to maintain $\mathrm{SpO}_{2}$ of $90-95 \%$ at and beyond $10 \mathrm{~min}$ of age (Figure 1).

\subsection{Outcomes}

The primary outcome was the feasibility to perform a cluster trial by changing local hospital policy for starting oxygen concentration and oxygen titration strategy. Feasibility is defined by (a) ability to achieve difference between the two groups in supplied oxygen concentration during initial resuscitation, and (b) ability to obtain deferred consent $>50 \%$ in infants who received the intervention. Secondary outcomes included: mortality prior to discharge from hospital, delivery room interventions (e.g., rate of intubation, rate of chest compression, use of epinephrine), mechanical ventilation, necrotizing enterocolitis, bronchopulmonary dysplasia (defined as oxygen and/or respiratory support at 36 weeks), retinopathy of prematurity, brain injury as indicated by abnormal cranial ultrasound. To quantify oxygen supplied over the first 5 and $10 \mathrm{~min}$ respectively, oxygen concentrations were added over the time period, representing a cumulative supplied oxygen concentration measure. For the first $5 \mathrm{~min}$, the minimum cumulative supplied oxygen concentration would therefore be $21 \% \times 5 \mathrm{~min}=105$, whereas the maximum total supplied oxygen would be $100 \% \times 5 \mathrm{~min}=500$. Correspondingly, for the first $10 \mathrm{~min}$, the minimum total supplied oxygen would be $21 \% \times 10 \mathrm{~min}=210$, whereas the maximum total supplied oxygen would be $100 \% \times 10 \mathrm{~min}=1000$.

\section{Statistical Analysis}

Data were analyzed as intention-to-treat and reported according to the CONSORTConsolidated Standards of Reporting Trials extension to randomized pilot and feasibility trials (17). Data were compared using Student's t-test for parametric and Mann-Whitney $\mathrm{U}$ test for nonparametric comparisons of continuous variables, and Fisher exact for categorical variables. The data are presented as mean (standard deviation (SD)) for normally distributed continuous variables and median (interquartile range (IQR)) when the distribution was skewed. $p$-values were 2 -sided and $p<0.05$ was considered statistically significant. Statistical analyses were performed with SPSS Statistics for Macintosh, Version 27.0 (IBM Corp, Armonk, NY, USA).

\section{Results}

A total of 34 infants ( $n=14,30 \% \mathrm{O}_{2}$ group; $n=20,60 \% \mathrm{O}_{2}$ group) were born during the study period. None were excluded for major congenital anomalies or decision not to resuscitate at birth. Four infants $\left(n=2,30 \% \mathrm{O}_{2}\right.$ group; $n=2,60 \% \mathrm{O}_{2}$ group) were excluded as parents declined consent after the study intervention had been performed. In the eight infants who died $(n=5)$ or were transferred to another hospital before parents could be approached for consent $(n=3)$, limited data were obtained (Figure 2). We achieved a consent rate of $63 \%$. A total of 30 infants $\left(n=12,30 \% \mathrm{O}_{2}\right.$ group; $n=18,60 \% \mathrm{O}_{2}$ group) were included in the final analysis (Figure 1). Demographics of included infants are presented in Table 1 . The study protocol was followed in 10/12 infants (83\%) and 13/18 infants $(72 \%)$ in $30 \% \mathrm{O}_{2}$ vs. $60 \% \mathrm{O}_{2}$ groups, respectively. All protocol deviations related to increasing oxygen concentration more quickly than specified in the protocol. Despite protocol deviations, we achieved a difference in median cumulative supplied oxygen concentration between the two intervention groups for the first $5 \mathrm{~min}$ (240 (IQR 170-270) in the $30 \% \mathrm{O}_{2}$ group vs. $315(285-375)$ in the $60 \% \mathrm{O}_{2}$ Group, $p=0.002$ ) (Figure 3). However, the median total supplied oxygen concentration for the first $10 \mathrm{~min}$ was similar between groups (522 in the $30 \% \mathrm{O}_{2}$ group vs. 561 in the $60 \% \mathrm{O}_{2}$ Group, $p=0.172$ ) Maximum oxygen 
concentration supplied in the first 10 min was also similar between groups ( $84 \%$ vs. $78 \%$, $p=0.117)$

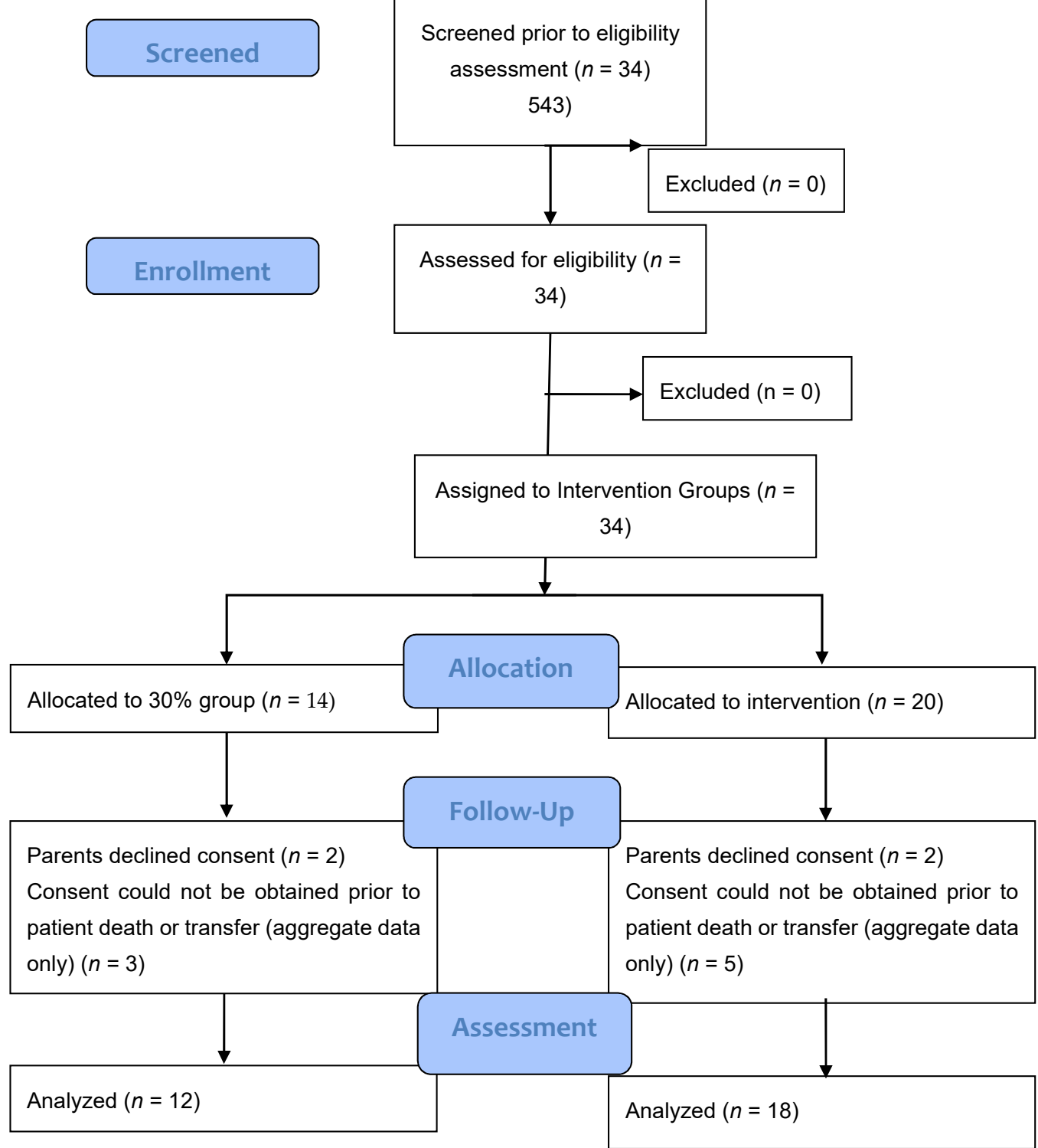

Figure 2. Consort-diagram.

Table 1. Demographics of study infants.

\begin{tabular}{cccc}
\hline & $\begin{array}{c}\mathbf{3 0} \% \text { Oxygen } \\
(\boldsymbol{n}=\mathbf{1 2})\end{array}$ & $\begin{array}{c}\mathbf{6 0} \% \text { Oxygen } \\
(\boldsymbol{n}=\mathbf{1 8})\end{array}$ & $\boldsymbol{p}$-Value \\
\hline Birth weight $(\mathrm{g})$ & $847(265)$ & $1000(247)$ & 0.127 \\
Gestational age (weeks) & $25(1.8)$ & $26(1.6)$ & 0.131 \\
Male $(n)$ * & $7(58 \%)$ & $6(35 \%)$ & 0.219 \\
Antenatal steroids $(n)^{*}$ & $11(92 \%)$ & $16(94 \%)$ & 1.00 \\
Apgar 1 min \# & $2(1-5)$ & $5(2-5)$ & 0.149 \\
Apgar 5 min $\#$ & $6(6-7)$ & $7(4-8)$ & 0.258 \\
Delayed Cord Clamping $(n) *$ & $5(42 \%)$ & $15(83 \%)$ & 0.045 \\
\hline
\end{tabular}

Data are presented as mean (SD), unless indicated ${ }^{\#}$ median (IQR), ${ }^{*} n(\%)$. 


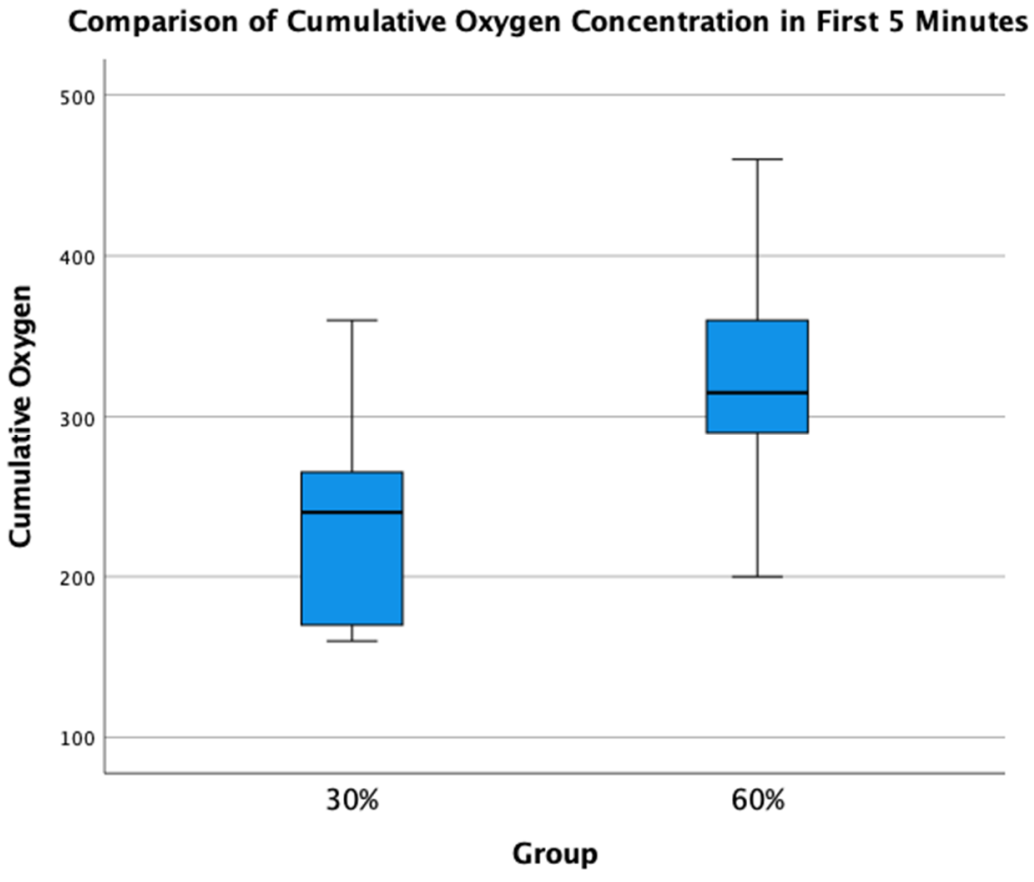

Figure 3. Comparison of Total Supplied Oxygen Concentrations for the first $5 \mathrm{~min}$.

In the $30 \%$ and $60 \% \mathrm{O}_{2}$ groups, $12(100 \%)$ and $17(94 \%)$ received positive pressure ventilation $(p=1.000)$; and $6(50 \%)$ and $6(33 \%)$ were intubated, respectively $(p=0.458)$. One infant in the $30 \% \mathrm{O}_{2}$ group received chest compression and epinephrine in the delivery room; this infant did not survive to admission to NICU. Infants in the $30 \% \mathrm{O}_{2}$ group had trend towards lower $\mathrm{SpO}_{2}$ by $5 \mathrm{~min}$ of age (53\% vs. $\left.71 \%, p=0.093\right)$ but similar mean heart rate (129 (29) vs. 119 (37) beats per minute, $p=0.47)$. Proportions of infants who had $\mathrm{SpO}_{2}<80 \%$ at 5 min were not statistically different $\left(9 / 11\right.$ in the $30 \% \mathrm{O}_{2}$ group vs. $9 / 18$ in the $60 \% \mathrm{O}_{2}$ group, $p=0.226$ ) (Figure 4 ).

The number of infants diagnosed with bronchopulmonary dysplasia was $6(67 \%)$ in the $30 \% \mathrm{O}_{2}$ group vs. $1(7 \%)$ in the $60 \% \mathrm{O}_{2}$ group $(p=0.0049)$. No other differences in secondary neonatal outcomes were observed (Table 2).

Table 2. Secondary neonatal outcomes.

\begin{tabular}{cccc}
\hline & $\begin{array}{c}\mathbf{3 0} \text { \% Oxygen } \\
(\boldsymbol{n}=\mathbf{1 2})\end{array}$ & $\begin{array}{c}\mathbf{6 0 \%} \text { Oxygen } \\
(\boldsymbol{n}=\mathbf{1 8})\end{array}$ & $\boldsymbol{p}$-Value \\
\hline Surfactant & $11(92 \%)$ & $13(72 \%)$ & 0.521 \\
Death before discharge & $3(25 \%)$ & $4(22 \%)$ & 1.000 \\
Intraventricular hemorrhage all grades & $7(58 \%)$ & $8(44 \%)$ & 0.701 \\
Intraventricular hemorrhage & $4(33 \%)$ & $4(22 \%)$ & 0.677 \\
grade $\geq 3$ & $6(50 \%)$ & $9(50 \%)$ & 1.000 \\
Patent ductus arteriosus & $2(17 \%)$ & $3(17 \%)$ & 1.000 \\
Necrotizing enterocolitis & $6(67 \%)$ & $1(7 \%)$ & 0.0049 \\
$\begin{array}{c}\text { Chronic lung disease in survivor } \\
\text { Retinopathy of prematurity } \\
\text { in survivor }\end{array}$ & $3(33 \%)$ & $3(27 \%)$ & 0.643 \\
\hline
\end{tabular}

Data are presented as $n(\%)$. 

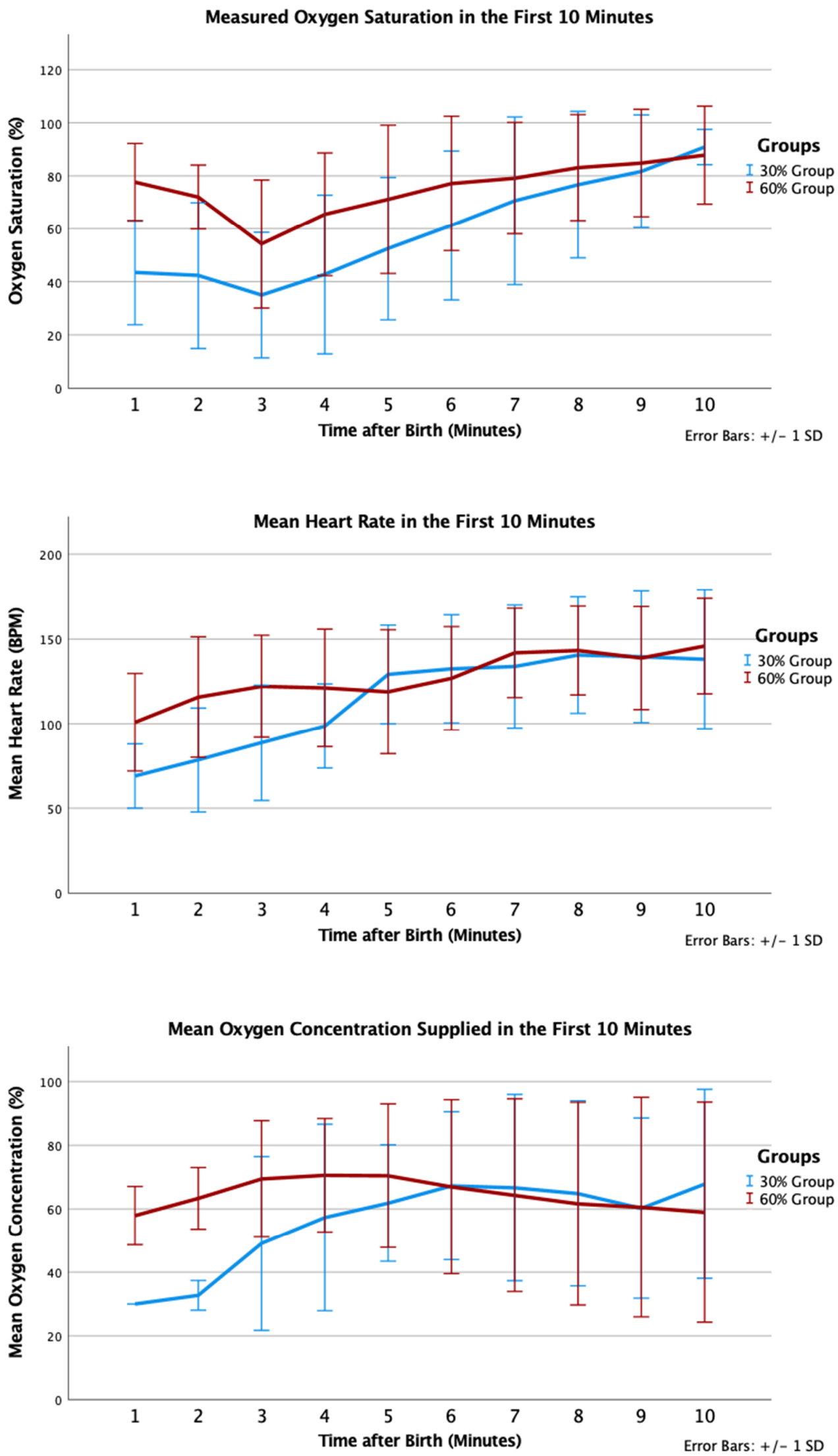

Figure 4. Mean oxygen saturation (\%), oxygen saturation (\%), and heart rate (bpm) in the first $10 \mathrm{~min}$ after birth according to group allocation. 


\section{Discussion}

Recent neonatal guidelines recommend a starting oxygen concentration between $21 \%$ to $30 \%$, based on very low-certainty evidence [6]. However, the optimal starting oxygen concentration for the resuscitation of extremely preterm infants remains unknown. Hyperoxia results in an increase in oxygen free radicals and decrease cerebral blood flow [ $8-10$ ], while oxygen saturation of $<80 \%$ at $5 \mathrm{~min}$ have been associated with increased mortality or neurodevelopmental disabilities [11,13].

A recent meta-analysis included 10 randomized trials and four cohort studies and demonstrated no significant risk or harm from either strategy [7]; however, included trials were small, with the largest trial being the To2rpido-trial with 287 patients [15]. In comparison, an individual patient meta-analysis analysis of eight trials $(n=768)$ reported that infants initially resuscitated with $21-30 \%$ vs. $\geq 60 \% \mathrm{O}_{2}$ were less likely to achieve $\mathrm{SpO}_{2} \geq 80 \%$, which was associated with increased risk of major intraventricular hemorrhage and five times higher risk of death [14]. Unfortunately, most recent Individual patient randomized trials, including To2rpido and PRESOX (NCT01773746), have ceased early due to low enrolment $[15,16]$.

Our study demonstrates the feasibility of using two starting oxygen concentrations for delivery room resuscitation of extremely preterm infants using deferred consent, mimicking single-center participation in a multi-center, cluster-randomized, crossover trial. We chose this design due to the often emergent nature of preterm births and the need to initiate immediate resuscitation in most situations. Consent was then obtained using a deferred consent model with written consent sought from the parents of these infants as soon as possible after birth to utilize data for research [19,20], as per the Canadian Tri-Council Policy Statement (TCPS) in Human Research guidelines. In Canada, TCPS policy explicitly sets out criteria allowing for "Exception to the requirement to seek prior consent", which include: (i) necessity to answer the research question, (ii) lack of adverse impact on participants, (iii) justification of individual or society benefits compared with risks, (iv) minimal risk of interventions. In addition, this policy stipulates that the lack of prior consent "may be addressed through debriefing conducted as soon as possible following participants' involvement in the research, and within a timeframe that allows participants to withdraw their data or biological materials" [21]. Additional criteria exist for altering the need for prior consent in emergency situations, where a potential participant requires immediate medical intervention, that there is no additional risk in the study intervention or even potential benefit, and that there is insufficient time to obtain consent from an authorized third party. These requirements for deferred consent differ between jurisdictions, which may complicate multi-national trials [20]. Using this approach, our consent rate was 63\%; therefore, the estimated recruitment of over 100 subjects could be projected over a recruitment period of one year at a center of our size assuming a similar rate of preterm deliveries and consent rate. This might overcome limitations in recruitment encountered when using an antenatal consent approach, as experienced by previous randomized trials comparing higher vs. lower initial oxygen; in the To2rpido study, only 292 out of 6291 eligible infants were enrolled (4.6\%) [15].

Another advantage to our approach is that infants born precipitously (where there is inadequate time for either antenatal consent or pre-delivery randomization) could be included, which could potentially increase the generalizability of results. An example of this was seen in the HIPSTER trial, where a change during the trial from prospective consent only to a mixed prospective/deferred consent strategy resulted in a larger proportion of eligible infants recruited and differences in infant demographics [22]. A mixed-methods study of parental opinions on deferred consent approach for a trial comparing delayed cord clamping vs. cord milking reported that this approach is highly acceptable for most families and might be preferable to prospective consent for some, specifically for interventions that are of low risk and within the standard of practice [23]. Parental perceptions of deferred consent are more mixed in trials examining interventions that are not considered low risk [23]. Our study interventions of using $30 \%$ vs. $60 \% \mathrm{O}_{2}$ can be considered low 
risk and falls within the range of $\mathrm{O}_{2}$ used by clinicians in the course of neonatal resuscitation. A combined prospective/deferred consent strategy, where antenatal consent is obtained when possible, could be considered. However, this mixed consent strategy may be difficult to implement for a cluster-randomized trial, as all eligible infants born during the trial period would otherwise follow study interventions unless a family opts to not participate antenatally. Finally, in contrast to trials comparing $21 \%$ vs. $100 \% \mathrm{O}_{2}$, a starting concentration of $30 \%$ vs. $60 \% \mathrm{O}_{2}$ might also be more acceptable to clinicians [5].

Our difference in starting oxygen concentration was less than that of previous trials, yet we achieved a difference in supplied oxygen concentration over the first $5 \mathrm{~min}$ using a combination of (i) different initial oxygen concentrations and (ii) changing oxygen titration strategy based on a combination of heart rate and time-based saturation targets. The 30\% $\mathrm{O}_{2}$ group had a lower mean $\mathrm{SpO}_{2}$ at 5 min compared with the $60 \% \mathrm{O}_{2}$ group (53\% vs. $71 \%, p=0.093$ ); however, this must be interpreted with caution as the $30 \%$ group had younger and smaller infants, lower rate of delayed cord clamping, and a lower proportion of female infants. Finally, both groups had a significant proportion of infants who did not achieve $\geq 80 \% \mathrm{SpO}_{2}$ by $5 \mathrm{~min}$, as oxygen was not adjusted before $5 \mathrm{~min}$ after birth Therefore, future trials should consider earlier and/or more rapid oxygen concentration increases to achieve this goal, given the association between $\mathrm{SpO}_{2}<80 \%$ at 5 min with brain injury and poor neurological outcomes.

\section{Limitations}

We acknowledge that the number of subjects is too small to draw any conclusions regarding the relative efficacy of the two different oxygen concentrations. While not statistically significant, the $60 \%$ group had a higher mean birth weight and a higher proportion of female infants, which could explain the lower rates of bronchopulmonary dysplasia. We are currently organizing a multi-center cluster-randomized trial comparing $30 \%$ vs. $60 \%$ oxygen (HiLo-NCT02858583) to study this in a larger patient population [24].

\section{Conclusions}

Using a change in local hospital policy with deferred consent in a cross-over design can achieve a difference in supplied oxygen in the first $5 \mathrm{~min}$ of resuscitation with acceptable consent rate, making this study design feasible for a larger, multi-centered cluster-randomized crossover trial to study whether $30 \%$ vs. $60 \%$ would be the optimal starting oxygen for resuscitation of infants $<29$ weeks' gestational age.

Author Contributions: Conception and design, G.M.S., N.N.F., M.V., W.T.-M., M.Y., P.S.S. and E.A.; data curation, G.M.S., N.N.F., M.V., W.T.-M., P.S.S., E.A. and B.H.Y.L.; Analysis and interpretation of the data, G.M.S., M.Y., N.N.F., M.V., W.T.-M., P.S.S., E.A. and B.H.Y.L.; Drafting of the article, G.M.S., N.N.F., M.V., W.T.-M., M.Y., P.S.S., E.A. and B.H.Y.L.; Critical revision of the article for important intellectual content, G.M.S., N.N.F., M.V., W.T.-M., M.Y., P.S.S., E.A. and B.H.Y.L.; Final approval of the article, G.M.S., N.N.F., M.V., W.T.-M., P.S.S., M.Y., E.A. and B.H.Y.L. All authors have read and agreed to the published version of the manuscript.

Funding: This research received no external funding.

Institutional Review Board Statement: The study was conducted according to the guidelines of the Declaration of Helsinki and approved by the Royal Alexandra Hospital Research Committee and Health Ethics Research Board, University of Alberta (Pro00084090) approved the study and the study was registered at Ciinicaltrials.gov (NCT03706586) on 18 October 2018.

Informed Consent Statement: Written informed consent for use of patient data was sought from the parents.

Data Availability Statement: All relevant data are in the manuscript.

Acknowledgments: We would like to thank the parents and infants agreeing to be part of the study. We would like to thank the Resuscitation-Stabilization-Triage team of the Royal Alexandra Hospital for helping and supporting the study. 
Conflicts of Interest: The authors declare no conflict of interest.

$\begin{array}{ll}\text { Abbreviations } \\ \mathrm{SpO}_{2} & \text { Oxygen saturation } \\ \mathrm{HR} & \text { Heart rate } \\ \mathrm{NICU} & \text { Neonatal Intensive Care Unit } \\ \mathrm{SD} & \text { Standard deviation } \\ \mathrm{IQR} & \text { Interquartile range }\end{array}$

\section{References}

1. $\quad$ East, C.E.; Colditz, P.B.; Begg, L.M.; Brennecke, S.P. Update on intrapartum fetal pulse oximetry. Aust. N. Z. J. Obstet. Gynaecol. 2002, 42, 119-124. [CrossRef] [PubMed]

2. Dawson, J.A.; Kamlin, C.O.F.; Vento, M.; Wong, C.; Cole, T.J.; Donath, S.M.; Davis, P.G.; Morley, C.J. Defining the reference range for oxygen saturation for infants after birth. Pediatrics 2010, 125, e1340-e1347. [CrossRef]

3. Kapadia, P.; Hurst, C.; Harley, D.; Flenady, V.; Johnston, T.; Bretz, P.; Liley, H.G. Trends in neonatal resuscitation patterns in Queensland, Australia-A 10-year retrospective cohort study. Resuscitation 2020, 157, 126-132. [CrossRef] [PubMed]

4. Kattwinkel, J.; Perlman, J.M.; Aziz, K.; Colby, C.; Fairchild, K.; Gallagher, J.; Hazinski, M.F.; Halamek, L.P.; Kumar, P.; Little, G.; et al. Part 15: Neonatal resuscitation: 2010 American Heart Association Guidelines for Cardiopulmonary Resuscitation and Emergency Cardiovascular Care. Circulation 2010, 122, S909-S919. [CrossRef] [PubMed]

5. $\quad$ Oei, J.L.; Ghadge, A.; Coates, E.; Wright, I.M.; Saugstad, O.D.; Vento, M.; Buonocore, G.; Nagashima, T.; Suzuki, K.; Hosono, S.; et al. Clinicians in 25 countries prefer to use lower levels of oxygen to resuscitate preterm infants at birth. Acta Paediatr. Int. J. Paediatr. 2016, 105, 1061-1066. [CrossRef] [PubMed]

6. Wyckoff, M.H.; Wyllie, J.; Aziz, K.; de Almeida, M.F.; Fabres, J.; Fawke, J.; Guinsburg, R.; Hosono, S.; Isayama, T.; Kapadia, V.S.; et al. Neonatal Life Support: 2020 International Consensus on Cardiopulmonary Resuscitation and Emergency Cardiovascular Care Science with Treatment Recommendations. Circulation 2020, 142, S185-S221. [CrossRef] [PubMed]

7. Welsford, M.; Nishiyama, C.; Shortt, C.; Weiner, G.; Roehr, C.C.; Isayama, T.; Dawson, J.A.; Wyckoff, M.H.; Rabi, Y. Initial oxygen use for preterm newborn resuscitation: A systematic review with meta-analysis. Pediatrics 2019, 143, e20181828. [CrossRef] [PubMed]

8. Vento, M.; Saugstad, O.D. Oxygen supplementation in the delivery room: Updated information. J. Pediatr. 2011, 158, e5-e7. [CrossRef] [PubMed]

9. Saugstad, O.D.; Ramji, S.; Vento, M. Oxygen for newborn resuscitation: How much is enough? Pediatrics 2006, 118, 789-792. [CrossRef] [PubMed]

10. Finer, N.; Saugstad, O.; Vento, M.; Barrington, K.; Davis, P.; Duara, S.; Leone, T.; Lui, K.; Martin, R.; Morley, C.; et al. Use of oxygen for resuscitation of the extremely low birth weight infant. Pediatrics 2010, 125, 389-391. [CrossRef] [PubMed]

11. Katheria, A.C.; Hassen, K.; Rich, W.; Poeltler, D.; Finer, N. Resuscitation outcomes of infants that do not achieve a 5 min target SpO2 saturation. J. Perinatol. 2019, 39, 1635-1639. [CrossRef] [PubMed]

12. Saugstad, O.D.; Aune, D.; Aguar, M.; Kapadia, V.; Finer, N.; Vento, M. Systematic review and meta-analysis of optimal initial fraction of oxygen levels in the delivery room at $\leq 32$ weeks. Acta Paediatr. Int. J. Paediatr. 2014, 103, 744-751. [CrossRef] [PubMed]

13. Oei, J.L.; Finer, N.N.; Saugstad, O.D.; Wright, I.M.; Rabi, Y.; Tarnow-Mordi, W.; Rich, W.; Kapadia, V.; Rook, D.; Smyth, J.P.; et al. Outcomes of oxygen saturation targeting during delivery room stabilisation of preterm infants. Arch. Dis. Child. Fetal Neonatal Ed. 2018, 103, F446-F454. [CrossRef] [PubMed]

14. Oei, J.L.; Vento, M.; Rabi, Y.; Wright, I.; Finer, N.; Rich, W.; Kapadia, V.; Aune, D.; Rook, D.; Tarnow-Mordi, W.; et al. Higher or lower oxygen for delivery room resuscitation of preterm infants below 28 completed weeks gestation: A meta-analysis. Arch. Dis. Child. Fetal Neonatal Ed. 2017, 102, F24-F30. [CrossRef] [PubMed]

15. Oei, J.L.; Saugstad, O.D.; Lui, K.; Wright, I.M.; Smyth, J.P.; Craven, P.; Wang, Y.A.; McMullan, R.; Coates, E.; Ward, M.; et al. Targeted Oxygen in the Resuscitation of Preterm Infants, a Randomized Clinical Trial. Pediatrics 2017, 139, e20161452. [CrossRef] [PubMed]

16. Bethesda (MD): National Library of Medicine (US). Identifier NCT01773746, Study of Room Air Versus 60\% Oxygen for Resuscitation of Premature Infants (PRESOX). Available online: https:/ / clinicaltrials.gov/ct2/show/NCT01773746 (accessed on 21 July 2021).

17. Eldridge, S.M.; Chan, C.L.; Campbell, M.J.; Bond, C.M.; Hopewell, S.; Thabane, L.; Lancaster, G.A.; Altman, D.; Bretz, F.; Campbell, M.; et al. CONSORT 2010 statement: Extension to randomised pilot and feasibility trials. BMJ 2016, 355 , i5239. [CrossRef] [PubMed]

18. Bethesda (MD): National Library of Medicine (US). Identifier NCT03706586, The Hilo Trial to Improve Neurodevelopmental Outcomes in Very Low Birthweight Infants-Pilot-Trial. Available online: https:/ / clinicaltrials.gov/ct2/show / NCT03706586 (accessed on 21 July 2021).

19. Gale, C.; Hyde, M.J.; Modi, N. Research ethics committee decision-making in relation to an efficient neonatal trial. Arch. Dis. Child. Fetal Neonatal Ed. 2017, 102, F291-F298. [CrossRef] [PubMed] 
20. Den Boer, M.C.; Houtlosser, M.; Foglia, E.E.; Davis, P.G.; Van Kaam, A.H.; Kamlin, C.O.F.; Schmölzer, G.M.; De Vries, M.C.; Te Pas, A.B. Deferred consent for the enrolment of neonates in delivery room studies: Strengthening the approach. Arch. Dis. Child. Fetal Neonatal Ed. 2019, 104, F348-F352. [CrossRef] [PubMed]

21. Canadian Institutes of Health Research; Natural Sciences and Engineering Research Council of Canada; Social Sciences and Humanities Research Council. Tri-Council Policy Statement: Ethical Conduct for Research Involving Humans-TCPS 2. Available online: https://ethics.gc.ca/eng/tcps2-eptc2_2018_introduction.html (accessed on 12 October 2017).

22. Songstad, N.T.; Roberts, C.T.; Manley, B.J.; Owen, L.S.; Davis, P.G. Retrospective consent in a neonatal randomized controlled trial. Pediatrics 2018, 141, e20172092. [CrossRef] [PubMed]

23. Rich, W.D.; Katheria, A.C. Waiver of consent in a trial intervention occurring at birth-how do parents feel? Front. Pediatr. 2017, 5, 56. [CrossRef] [PubMed]

24. Bethesda (MD): National Library of Medicine (US). Identifier NCT03825835, 30\% or 60\% Oxygen at Birth to Improve Neurodevelopmental Outcomes in Very Low Birthweight Infants (HiLo). Available online: https:/ / clinicaltrials.gov/ct2/show / NCT03825835 (accessed on 21 July 2021). 